\title{
QUERCETIN AND RUTIN: EFFECTS OF TWO FLAVONOIDS ON INDUCED OXIDATIVE STRESS
}

\section{IN HUMAN EJACULATED SPERM}

Mazzi L. ${ }^{1}$, Geminiani M. ${ }^{1}$, Collodel G. ${ }^{1,2}$, Iacoponi F. ${ }^{1}$, Martini S. ${ }^{3,4}$, Bonechi C. ${ }^{3,4}$, Rossi C. ${ }^{3,4}$, Moretti E. ${ }^{1,2}$

\author{
${ }^{1}$ Department of Biomedical Sciences, Applied Biology Section, University of Siena \\ ${ }^{2}$ Interdepartmental Centre for Research and Therapy of Male Infertility, University of Siena \\ ${ }^{3}$ Department of Pharmaceutical and Applied Chemistry, University of Siena, Italy \\ ${ }^{4}$ Centre for Colloid and Surface Science (CSGI), University of Florence, Italy
}

\begin{abstract}
Quercetin (Q) and rutin (R) are two natural flavonoids with antioxidant properties. We evaluated the toxicity of $\mathrm{Q}$ and $\mathrm{R}$ at $20 \mu \mathrm{M}, 30 \mu \mathrm{M}, 50 \mu \mathrm{M}, 100 \mu \mathrm{M}, 200 \mu \mathrm{M}, 400 \mu \mathrm{M}$ in swim up selected human sperm. The antioxidant activity $(\mathrm{Q}$ and $\mathrm{R} 20 \mu \mathrm{M}$ and $30 \mu \mathrm{M}$ ) was tested on lipid peroxidation (LPO) induced by tert-butylhydroperoxide in human sperm. LPO was evaluated using the C11-BODIPY581/591 probe and sperm structural damages were assessed by transmission electron microscopy in samples incubated with and without $\mathrm{Q}$ and R. A significant dose dependent toxic effect was observed for both compounds on sperm viability $(\mathrm{Q}$ and $\mathrm{R}: \mathrm{r}=-0.98 \mathrm{P}<0.001)$, on sperm progressive motility $(\mathrm{Q}: \mathrm{r}=-0.98, \mathrm{R}: \mathrm{r}=-0.97$; $\mathrm{P}<0.001)$, and on non progressive motility $(\mathrm{Q} ; \mathrm{r}=-0.58, \mathrm{P}<0.01$; R: $\mathrm{r}=-0.50, \mathrm{P}<0.05)$. Both flavonoids, used at $20 \mu \mathrm{M}$ and $30 \mu \mathrm{M}$, showed antioxidant properties on LPO induced in human sperm and a general protective effect against ultrastructural damages of LPO. In conclusion, we observed that $\mathrm{Q}$ exhibited a little higher toxicity than $\mathrm{R}$; on the other hand $\mathrm{R}$ is little low protective on induced LPO. Our preliminary results demonstrated the scavenger properties of these flavonoids in vitro on human sperm. Keywords: human sperm, lipid peroxidation, quercetin, rutin, TEM.
\end{abstract}

\section{INTRODUCTION}

Oxidative stress is a condition that is associated with an imbalance between the production and the removal of reactive species and free radicals by antioxidants. Reactive oxygen species (ROS) are normal products of cellular metabolism and they play a functional role in many cell types. When ROS are produced at a very low concentration, they trigger cell signaling events and regulate physiological function [1, 2].

Sperm were the first type of cell reported to generate free radicals [3]; low level production of ROS by sperm supports some main functions, such as capacitation, acrosome reaction, zona pellucida binding and oocyte fusion [4]. Nevertheless, uncontrolled ROS production can play an important role in causing sperm aberration, leading to infertility. Oxidative stress has been suggested to be an important factor in the aetiology of poor sperm function through peroxidative damage to the cell membrane, to DNA (inducing single- and doublestrand DNA breaks) and to proteins [5-7] and an excess of ROS and free radical generation has frequently been detected in the seminal plasma and the sperm of infertile men [8-10].

Seminal plasma and sperm are endowed with an array of protective antioxidants, such as the glutathione peroxidase/reductase system, superoxide dismutase, catalase and low-molecular weight antioxidants, vitamin
E, vitamin C, urate, and albumin, which scavenge ROS in order to prevent possible cellular damage [11]. One of the rational strategies for counteracting oxidative stress is to increase the scavenging capacity of seminal plasma.

The use of antioxidant supplementation to reverse the effect of ROS is still being debated [2, 12]. Exogenous antioxidants are substances introduced through the diet; the most important are vitamin E, vitamin C, beta carotene, coenzyme Q10, flavonoids.

Flavonoids are secondary plant metabolites and, during the last decades, have been under extensive investigation because of their claimed antioxidant, anti-inflammatory, antiallergic, antidiabetic, cardio, hepatio- and gastroprotective, antiviral and antineoplastic properties [13].

Among flavonoids we focused on quercetin (Q) and rutin $(R)$. Q, a reddish pigment, is found in many different foods, including in the pigments of the apple skins, in red wine and in red onions. Q has been largely investigated for its free radical scavenging and metal chelating abilities [14-16]. Particularly, the presence of three hydroxyl groups makes the compound a potential free radical scavenger and/or antioxidant. $\mathrm{R}$ is the glycoside of $\mathrm{Q}$ with 3-hydroxyl group blocked by a rutinoside group [17-18]; it is a bioflavonoid, abundantly present in onions, apples, tea and red wine 
[19]. A wide variety of biological activities of $R$, including antioxidant, has been reported [20].

The aim of this study was to investigate the antioxidant properties of Q and R against lipid peroxidation (LPO) induced in human ejaculated sperm by tert-butylhydroperoxide (TBHP). Furthermore, the possible citotoxicity of these compounds on sperm motility and viability was explored.

\section{MATERIALS AND METHODS}

\section{Semen analysis}

Semen samples were obtained from men recruited at the Interdepartmental Centre for Research and Therapy of Male Infertility, University of Siena. Semen samples were collected by masturbation after 3-5 days of sexual abstinence and examined after liquefaction for $30 \mathrm{~min}$ at $37^{\circ} \mathrm{C}$. Volume, $\mathrm{pH}$, concentration and motility were evaluated according to World Health Organization [21] guidelines. Patients were informed of and gave written consent for the procedures related to the study.

\section{Sperm selection: swim-up}

Motile sperm fraction was selected by the swim-up technique. A direct swim-up of sperm from semen was performed: $1 \mathrm{ml}$ of each semen sample was placed in a sterile conical centrifuge tube and gently layered with $1.2 \mathrm{ml}$ of Quinn's ${ }^{\circledR}$ Sperm Washing Medium (Sage, In vitro fertilization, Inc., Trumbull, CT, USA). The tubes, inclined at an angle of $45^{\circ}$, were incubated for $45 \mathrm{~min}$ at $37^{\circ} \mathrm{C}$ with $5 \% \mathrm{CO}_{2}$. The upper most $\mathrm{lml}$ of medium was then recovered, which contained highly motile sperm cells.

\section{$Q$ and $R$ preparation}

$\mathrm{Q}$ and $\mathrm{R}$ were purchased from Sigma-Aldrich Chemie GmbH (Buchs, Switzerland) and used without further purification. Stock solutions of $\mathrm{Q}$ and $\mathrm{R}$ at $400 \mu \mathrm{M}$ were prepared by dissolving these compounds in Biggers Whitten Whittingham (BWW; WHO, 2010). Little amount of $0.1 \mathrm{M} \mathrm{NaOH}$ solution were added to help dissolve each solution. The stock solutions were stored at $-20{ }^{\circ} \mathrm{C}$.

\section{Determination of sperm viability and motility after $Q$ and $R$ treatment}

The swim-up selected sperm were added to $20 \mu \mathrm{M}$, $30 \mu \mathrm{M}, 50 \mu \mathrm{M}, 100 \mu \mathrm{M}, 200 \mu \mathrm{M}, 400 \mu \mathrm{M}$ of $\mathrm{Q}$ and R stock solutions diluted in BWW medium. Mixtures were incubated at $37{ }^{\circ} \mathrm{C}$ and $5 \% \mathrm{CO}_{2}$ for $1 \mathrm{~h}$. The sperm were stained with $0.5 \%$ Eosin Y (CI 45380) in $0.9 \%$ aqueous sodium chloride solution and observed by light microscope (Leica, Wetzlar, Germany). Stained (dead) cells and unstained (viable) cells were scored. Sperm motility was evaluated in swim-up selected sperm incubated with $\mathrm{Q}$ and $\mathrm{R}$, using a Burker counting chamber and categorizing them by the different grades of motility (progressive motility, non progressive motility, and immotile sperm, [21]).

Aliquots of sperm devoid of $\mathrm{Q}$ and $\mathrm{R}$ and treated in the same conditions were used as the controls.

All experiments were carried out in triplicate and the results are reported as mean values and standard deviations.

\section{LPO induction and $Q / R$ treatment}

Selected human sperm were incubated with $5 \mu \mathrm{M}$ of a fluorescent fatty acid probe, 4,4-difluoro-5-(4-phenyl1,3-butadienyl)-4-bora-3a,4-adiaza-s-indacene-3-undecanoic acid (C11-BODIPY581/591, Molecular Probes, Eugene, USA) at $37{ }^{\circ} \mathrm{C}$ for $30 \mathrm{~min}$.

Excess probe was removed by washing the cells twice with BWW medium for 10 min. C11-BODIPY581/591 is able to intercalate in the phospholipid bilayer. The intact probe fluoresces red when it is intercalated into the membrane $(\lambda$ excitation $=590$ and $\lambda$ emission $=635$ $\mathrm{nm})$ and shifts to green $(\lambda$ excitation $=485$ and $\lambda$ emission $=535 \mathrm{~nm}$ ) after oxidative radicals attack.

After incubation with this probe, each sample was then divided into aliquots as follows: sperm treated with TBHP $(45 \mu \mathrm{M})$ and $\mathrm{Q}(20 \mu \mathrm{M}$ and $30 \mu \mathrm{M})$ or R $(20 \mu \mathrm{M}$ and $30 \mu \mathrm{M}$ ) and sperm treated with TBHP without Q/R. Aliquots were incubated at $37^{\circ} \mathrm{C}$ and $5 \% \mathrm{CO}_{2}$ for $1 \mathrm{~h}$. The staining was examined by Leitz Aristoplan Microscope fluorescence (Leica, Wetzlar, Germany). Images were acquired using Leica Q Fluoro Standard, Leica Chantal software. The presence of the green signal, a marker of LPO, was estimated in two degrees: high and low fluorescence. For each sperm sample 300 cells were examined. All experiments were carried out in triplicate and the results are reported as mean values and standard deviations.

\section{Transmission electron microscopy (TEM)}

Human sperm treated with TBHP and Q $(20 \mu \mathrm{M}$ and $30 \mu \mathrm{M})$ or $\mathrm{R}(20 \mu \mathrm{M}$ and $30 \mu \mathrm{M})$ and with TBHP without $\mathrm{Q}$ or R were processed for TEM. Sperm samples were fixed in cold Karnovsky fixative and maintained at 4 - $\mathrm{C}$ for $2 \mathrm{~h}$ and then washed in $0.1 \mathrm{~mol} / \mathrm{l}$ cacodylate buffer ( $\mathrm{pH} 7.2$ ) for $12 \mathrm{~h}$, postfixed in $1 \%$ buffered osmium tetroxide for $1 \mathrm{~h}$ at $4{ }^{\circ} \mathrm{C}$, dehydrated and embedded in Epon Araldite. Ultra-thin sections were cut with a Supernova ultramicrotome (Reickert Jung, Vienna, Austria), mounted on copper grids, stained with uranyl acetate and lead citrate and observed and photographed with a Philips EM208 transmission electron microscope (TEM; Philips Scientifics, Eindhoven, The Netherlands). A minimum of 300 sperm sections were analysed for each sample and the anomalies related to the acrosome (reacted, swollen), the chromatin (disrupted), the axoneme (disorganized) and the plasma membrane (broken) were quantified. These experiment were performed in triplicate.

\section{Statistical analysis}

The correlations between $\mathrm{Q}$ and $\mathrm{R}$ concentrations and the sperm viability and motility percentages were evaluated by Spearman correlation coefficient (r). A P $<0.05$ (two-tailed) was considered statistically significant. All analyses were performed using the SPSS statistical software ver. 16 (SPSS Inc., Chicago, Illinois, USA). 


\section{RESUlTS}

Determination of sperm viability and motility after $Q$ and $R$ treatment

The percentages of viable sperm incubated with different concentrations of $\mathrm{Q}$ and $\mathrm{R}$ are reported in Figure 1 .

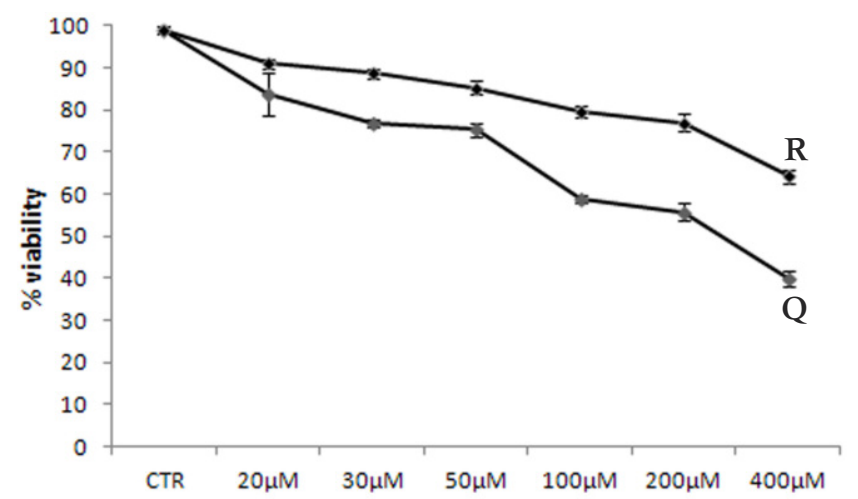

Figure 1. Mean of the percentages of viable human sperm treated with $Q$ and $R$ at different concentrations. $C T R=$ controls, same conditions without flavonoid

A significant dose dependent effect on sperm viability was observed for all the assayed compounds: the viability decreased significantly at the increase of $\mathrm{Q}(\mathrm{r}=-$ $0.98, \mathrm{P}<0.001)$ and $\mathrm{R}(\mathrm{r}=-0.98, \mathrm{P}<0.001)$ concentrations. In general, $Q$ showed a toxic effect on sperm viability higher than that exerted by $\mathrm{R}$.

In addition to viability, the effects of the different concentrations of $\mathrm{Q}$ and $\mathrm{R}$ on the progressive and non progressive motility of swim-up selected sperm were explored. Both flavonoids showed a dose dependent effect on sperm motility (Figure 2a, b). In particular, the percentage of sperm with progressive motility significantly decreased with the increase of $Q(r=-0.98$, $\mathrm{P}<0.001)$ and $\mathrm{R}(\mathrm{r}=-0.97, \mathrm{P}<0.001)$ concentrations (Figure $2 a)$. At the same concentrations $(\geq 50 \mu \mathrm{M})$ of used compounds, samples treated with $Q$ showed decreased progressive motility than those incubated with $\mathrm{R}$.
A dose dependent effect between the percentage of sperm with non progressive motility and the considered compounds was also observed (Figure 2b): the percentage of sperm with non progressive motility significantly decreased with the increase of $\mathrm{Q}(\mathrm{r}=-0.58$, $\mathrm{P}<0.01)$ and $\mathrm{R}(\mathrm{r}=-0.50, \mathrm{P}<0.05)$ concentrations.

\section{Effect of $Q$ and $R$ on induced LPO}

In human sperm LPO was induced by $45 \mu \mathrm{M}$ TBPH incubation; the effects of this treatment and of the scavenging power of $\mathrm{Q}$ and $\mathrm{R}$ were highlighted with the C11-BODIPY581/591 probe and TEM. For these experiments we used the concentrations that did not compromise sperm motility and viability: $20 \mu \mathrm{M}$ and $30 \mu \mathrm{M}$ of $\mathrm{Q}$ and $\mathrm{R}$.

\section{Detection with C11-BODIPY581/591 probe}

In human sperm, the localization of LPO was not equally distributed over the sperm membrane, but occurred mainly in the mid-piece region. The percentages of sperm with high intensity fluorescence (Figure 3), the marker of LPO, were significantly lower in samples treated with Q and R (Figures 4 B, C, E, F) compared to that observed in samples without $\mathrm{Q}$ and $\mathrm{R}$ (Figures 4 A, D).

\section{Detection of ultrastructural characteristics by TEM}

Ultrastructural characteristics of sperm samples treated with TBHP, both with and without $\mathrm{Q}$ and $\mathrm{R}$, were analysed by TEM. The percentages of the most frequent alterations of the acrosome, chromatin, axoneme and plasma membrane observed in these specimens are reported in Figure 5.

The vast majority of sperm treated with TBHP showed broken plasma membranes, reacted or swollen acrosomes, disrupted chromatin and tails with disorganized axoneme (Figure $6 \mathrm{~A}$ ).

Both $\mathrm{Q}$ and $\mathrm{R}$ showed a general protective effect against ultrastructural damages induced by LPO (Figures 6 B - E). However, Q exhibited a greater protective action than $\mathrm{R}$ particularly evident at acrosomal and plasma membrane level (Figures 6 B, C).

Figure $2(a-b)$. Mean of the percentages of progressive (a) and non progressive (b) motility (WHO, 2010) of human sperm treated with $Q$ and $R$ at different concentrations. CTR = controls, same conditions without flavonoid

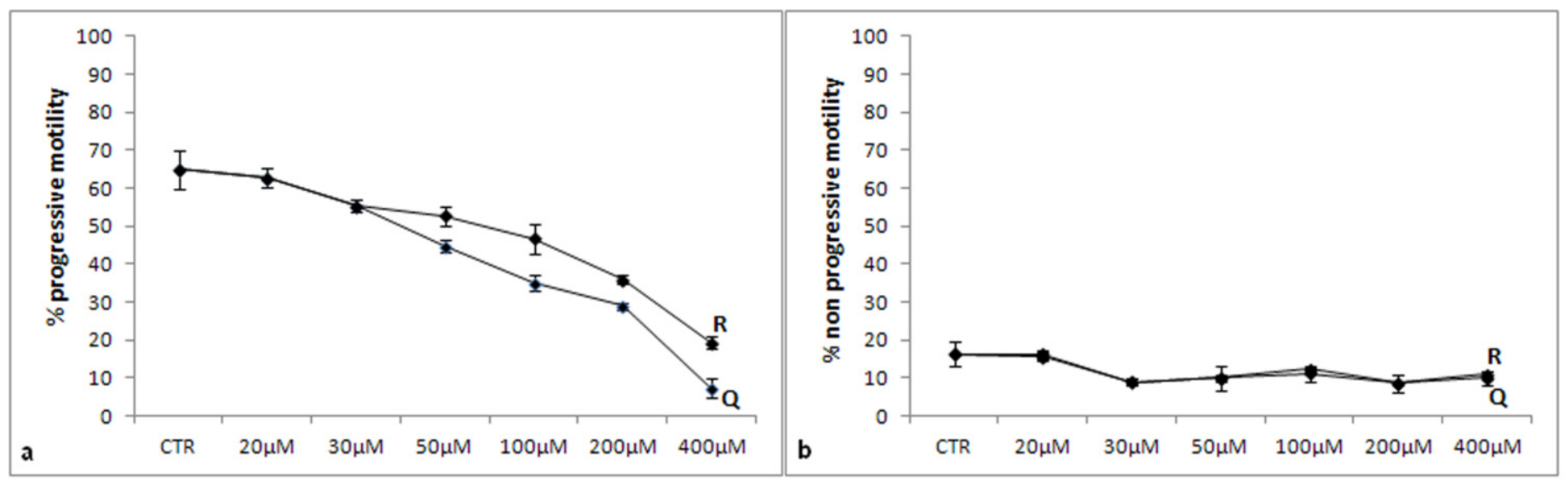




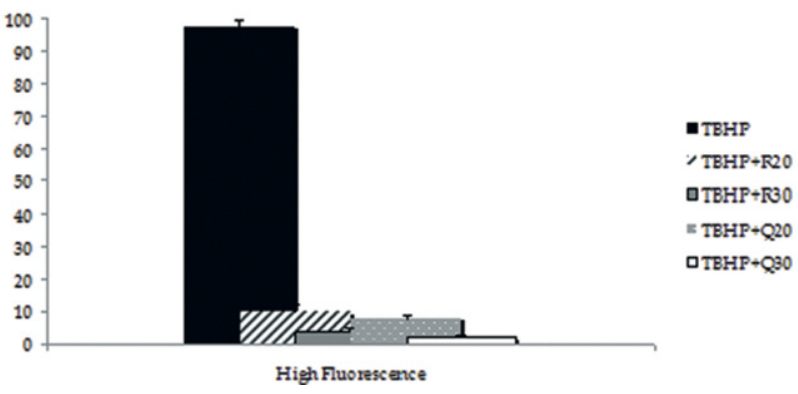

Figure 3. Means and standard deviation in the percentages of human sperm with a high level fluorescence (C11-BODIPY581/591 probe) indicating LPO induced by TBHP. Human sperm were treated with TBHP and Q20 or Q30 or R20 or $R 30$ and with TBHP only.

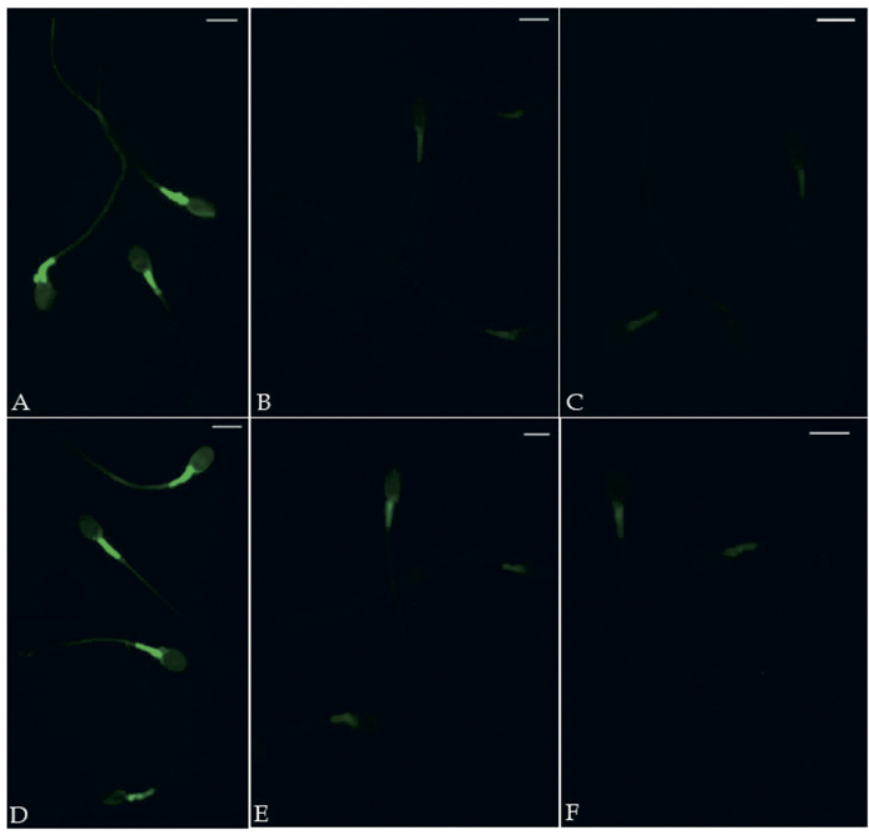

Figure 4. (A-F) Ultraviolet micrographs of human sperm treated with TBHP without $Q / R(A / D)$ and TBHP with $Q / R$.

C11-BODIPY581/591 probe was used to highlight LPO.

Sperm incubated with TBHP $(A, D)$. Sperm treated with $T B H P+Q 20(B), T B H P+Q 30(C), T B H P+R 20(E) e$ $T B H P+R 30(F) . B a r=5 \mu m$

\section{Discussion}

It is known that oxidative stress is a pathology detected in approximately half of all infertile men [2] which occurs when the complex of natural antioxidant defenses is jeopardized due to excessive ROS production. In the past decade, the interest in the bioactivities of flavonoids, mainly the antioxidant activity, on human health has been increased. As regards the reproductive field, whereas $\mathrm{Q}$ has been demonstrated to play a beneficial effect in male system of animal models exposed to oxidative stress [22-25], the possible antioxidant properties of $\mathrm{R}$ are still unexplored as well as the study performed in human semen samples.

In this paper we focused our attention on antioxidant effect of Q and R on LPO induced in vitro in a selected motile human sperm population. First of all, the potential cytotoxic effects of $Q$ and $R$ were investigated in order to choose appropriated $\mathrm{Q}$ and R concentrations $(20 \mu \mathrm{M}$ and $30 \mu \mathrm{M})$ to be used as antioxidant and able to preserve sperm motility and viability. We demonstrated that both compounds showed a protective and ROS scavenging actions in sperm cells incubated with TBHP, inductor of LPO. In particular, $Q$ showed a double faced action; on one hand it showed increased toxicity on sperm motility and viability than its glycoside $R$, on the other hand the antioxidant activity is little stronger than that exhibited by $\mathrm{R}$ and it was evident by both fluorescence analysis and TEM examination. A similar behavior of $\mathrm{Q}$ on sperm viability and motility was observed also by Khanduja et al. [26] which hypothesized an interaction between $\mathrm{Q}$ and a Ca2+ATPase, a key enzyme involved in the regulation of sperm motility. The little different activities of the two flavonoids on sperm motility, viability and antioxidant properties, could be explained by the different chemical structure of the two considered molecules. R shows the 3-hydroxyl group replaced with rutinose, a disaccharide consisting of glucose and rhamnose, which would decrease the capacity of $\mathrm{R}$ to penetrate through the plasma membrane; for this reason $\mathrm{R}$ appeared to be less harmful for sperm

Figure 5. Mean and standard deviation of the percentages of the most frequent alterations of acrosome, chromatin, axoneme,

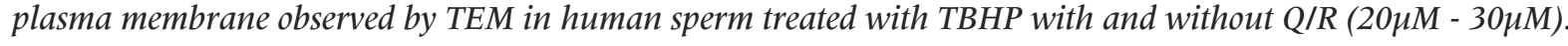

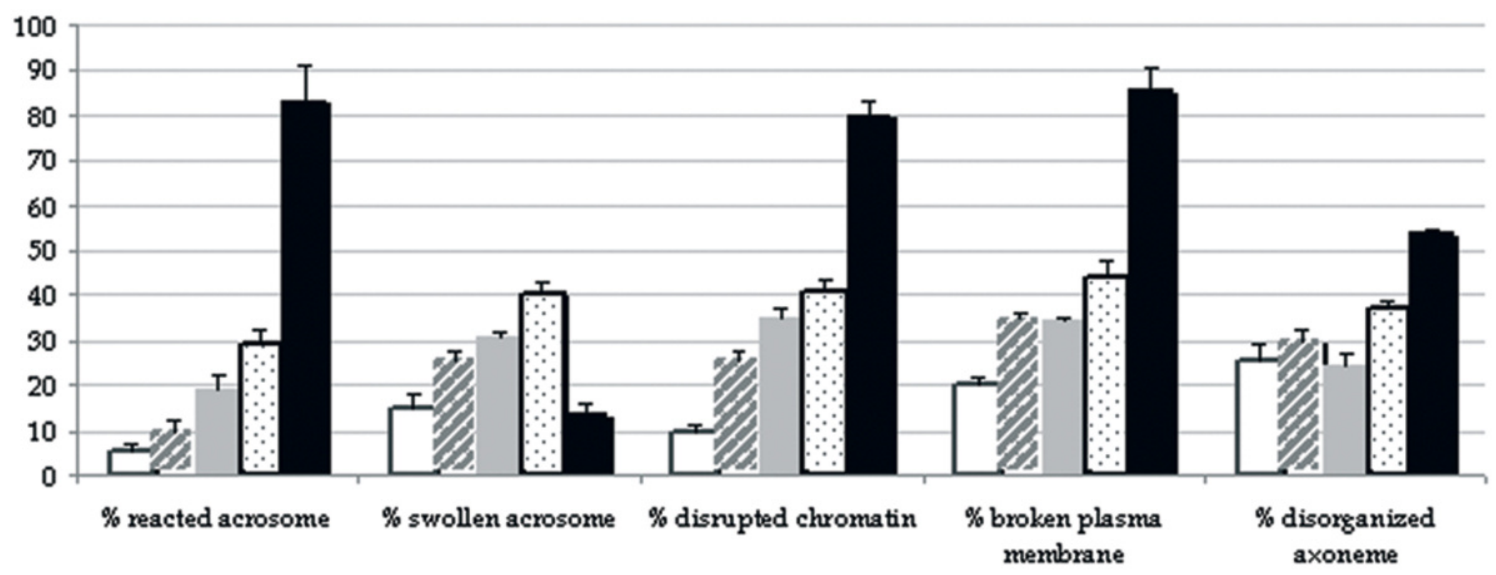

口 $\mathrm{TBHP}+\mathrm{Q} 30$

" $\mathrm{TBHP}+\mathrm{Q} 20$

$=\mathrm{TBHP}+\mathrm{R} 30$

- $\mathrm{TBHP}+\mathrm{R} 20$

- TBHP 


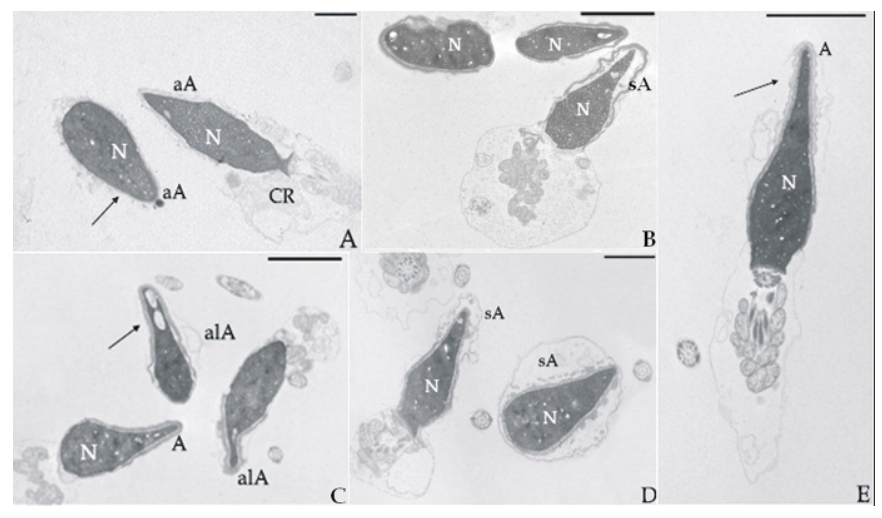

Figure $4(A-E)$. TEM micrographs of human sperm treated with TBHP without $Q / R$ and TBHP with $Q / R$. (A) Sperm incubated with TBHP; (B) Sperm incubated with TBHP+Q20; (C) Sperm incubated with TBHP+Q30; (D) Sperm incubated with TBHP+R20; (E) Sperm incubated with TBHP+R30. A: normal acrosome; aA: absent acrosome; alA: altered acrosome; $s$ : swollen acrosome; $N$ : nucleus; CR: cytoplasmic residue; arrow: broken membrane. Bars: $A, D=1 \mu m ; B, C, E=3 \mu m$.

viability and motility. The results of various in vitro and in vivo investigations strongly suggest a structureactivity relationship in the ability of flavonoids to act as antioxidants [27].

\section{Conclusion}

In conclusion, our results, although preliminary, support the evidence of the protective and antioxidant action of $\mathrm{Q}$ and $\mathrm{R}$ on human sperm cells incubated with TBHP. The development of new sperm culture media that can better protect sperm from ROS damage is required [2, 28, 29] and Q and R might be used as a ROS scavenger in such media, particularly in those used for mechanical techniques, such as IVF-ICSI or for cryopreservation of semen, in which oxidative stress is exacerbated. These studies obviously cannot be extrapolated to in vivo outcome, since the effect of $Q$ and $\mathrm{R}$ supplementation on male infertility has not yet been explored. To translate such findings into clinical reality, studies on the potential toxicity, pharmacokinetics and bioavailability of $\mathrm{Q} / \mathrm{R}$ in humans, followed by interventional clinical trials, are clearly needed.

\section{REFERENCES}

1. Forman, H.J.; Torres, M. Reactive oxygen species and cell signaling: respiratory burst in macrophage signaling. Am. J. Respir. Crit. Care Med. 166:4-8; 2002.

2. Tremellen, K. Oxidative stress and male infertility a clinical perspective. Hum. Reprod. Update 14:243-258; 2008.

3. MacLeod, J. The role of oxygen in the metabolism and motility of human spermatozoa. Am. J. Physiol. 138:512-518; 1943.

4. De Lamirande, E.; Jiang, H.; Zini, A.; Kodama, H.; Gagnon, C. Reactive oxygen species and sperm physiology. Rev. Reprod. 2:48-54; 1997. 5. Stadtman, E.R.; Levine, R.L. Free radical-mediated oxidation of free amino acids and amino acid residues in proteins. Amino Acids 25:207218; 2003.

6. Moustafa, M.H.; Sharma, R.K.; Thornton, J.; Mascha, E.; AbdelHafez, M.A.; Thomas, A.J. Jr; Agarwal, A. Relationship between ROS production, apoptosis and DNA denaturation in spermatozoa from patients examined for infertility. Hum. Reprod. 19:129-138; 2004.

7. Aitken, R.J.; De Luliis, G.N. Value of DNA integrity assays for fertility evaluation. Soc. Reprod. Fertil. Suppl. 65:81-92; 2007.

8. Nakamura, H; Kimura, T.; Nakajima, A.; Shimoya, K.; Takemura, M.; Hashimoto, K.; Isaka, S.; Azuma, C.; Koyama, M.; Murata, Y. Detection of oxidative stress in seminal plasma and fractionated sperm from subfertile male patients. Eur. J. Obstet. Gynecol. Reprod. Biol. 105:155$160 ; 2002$.

9. Baker, M.A.; Aitken, R.J. Reactive oxygen species in spermatozoa: methods for monitoring and significance for the origins of genetic disease and infertility. Reprod. Biol. Endocrinol. 3:67; 2005.

10. Pasqualotto, F.F.; Sharma, R.K.; Pasqualotto, E.B.; Agarwal, A. Poor semen quality and ROS-TAC scores in patients with idiopathic infertility. Urol. Int. 81:263-270; 2008.

11. Smith, R.; Vantman, D.; Ponce, J.; Escobar, J.; Lissi, E. Total antioxidant capacity of human seminal plasma. Hum. Reprod. 11:1655-1660; 1996.

12. Agarwal, A.; Nallella, K.P.; Allamaneni, S.S.; Said, T.M. Role of antioxidants in treatment of male infertility: an overview of the literature. Reprod. Biomed. Online 8:616-627; 2004.

13. Yao, L.H.; Jiang, Y.M.; Shi, J.; Tomàs-Barberàn, F.A.; Datta, N.; Singanusong, R.; Chen, S.S.; Flavonoids in food and their health benefits. Plant Foods Hum. Nutr. 59:113-122; 2004.

14. Jovanovic, S.V.; Steenkenm, S.; Tosic, M.; Marjanovic, B.; Simic, M.G. Flavonoids as antioxidants. J. Am. Chem. Soc. 116:4846-4851; 1994.

15. Terao, J.; Piskula, M.; Yao, Q. Protective effects of epicatechin, epicatechin gallate and quercetin on lipid peroxidation in phospholipid bilayers. Arch. Biochem. Biophys. 308:278-284; 1994.

16. Hu, J.P.; Calomme, M.; Lasure, A.; De Bruyne, T.; Pieters, C.; Ulietinck, A.; Van den Berghe, D.A. Structure activity relationships of flavonoids with superoxide scavenging ability. Biol. Trace Elem. Res. 47:327-331; 1995.

17. Rice-Evans, C.A.; Miller, N.J.; Paganga, G. Structure-antioxidant activity relationships of flavonoids and phenolic acids. Free Rad. Biol. Med. 20:933-956; 1996.

18. Lien, E.J.; Ren, S.; Bui, H.H.; Wang, R. Quantitative structure-activity relationship analysis of phenolic antioxidants. Free Rad. Biol. Med. 26:285-294; 1999

19. Hertog, M.G.; Hollman, P. C.; Katan, M.B.; Kromhout, D. Intake of potentially anticarcinogenic flavonoids and their determinants in adults in The Netherlands. Nutr. Canc. 20:21-29; 1993.

20. Janbaz, K. H.; Saeed, S. A.; Gilani, A. H. Protective effect of rutin on paracetamol- and CCl4-induced hepatotoxicity in rodents. Fitot. 73:557563: 2002.

21. World Health Organization (WHO) Laboratory Manual for the Examination and Processing of Human semen. Cambridge: Cambridge University; 2010.

22. Khaki, A.; Fathiazad, F.; Nouri, M.; Khaki, A.; Maleki, N.A.; Khamnei, H.J.; Ahmadi, P. Beneficial effects of quercetin on sperm parameters in streptozotocin-induced diabetic male rats. Phytother. Res. 24:1285$1291 ; 2010$.

23. Ben Abdallah, F.; Fetoui, H.; Zribi, N.; Fakhfakh, F.; Keskes, L. Quercetin attenuates lambda cyhalothrin-induced reproductive toxicity in male rats. Environ. Toxicol. DOI: 10.1002/tox.20762; 2011.

24. Ben Abdallah, F.; Zribi, N.; Ammar-Keskes, L. Antioxidative potential of Quercetin against hydrogen peroxide induced oxidative stress in spermatozoa in vitro. Andrologia 43:261-265; 2011.

25. Ciftci, O.; Aydin, M.; Ozdemir, I.; Vardi, N. Quercetin prevents 2,3,7,8tetrachlorodibenzo-p-dioxin-induced testicular damage in rats. Andrologia, 44:164-173, 2012, doi: 10.1111/j.1439 0272.2010.01126.x.; 2011. 26. Khanduja, K.L.; Verma, A.; Bhardwaj, A. Impairment of human sperm motility and viability by quercetin is independent of lipid peroxidation. Andrologia 33:277-281; 2001.

27. Metodiewa, D.; Kochman, A.; Karolczak, S. Evidence for antiradical and antioxidant properties of four biologically active $N, N$ - diethylaminoethyl ethers of flavonone oximes: a comparison with natural polyphenolic flavonoid (rutin) action. Biochem. Mol. Biol. Int. 41:10671075: 1997.

28. Garcez, M.E.; Branco, C.D.; Lara, L.V.; Pasqualotto, F.F.; Salvador, $M$. Effects of resveratrol supplementation on cryopreservation medium of human semen. Fertil. Steril. 94:2118-2121; 2010.

29. Collodel, G.; Federico, M.G.; Geminiani, M.; Martini, S.; Bonechi, C.; Rossi, C.; Figura, N.; Moretti, E. Effect of trans-resveratrol on induced oxidative stress in human sperm and in rat germinal cells Reprod. Toxicol. 31:239-246; 2011. 